\title{
Micropropagation of Pecan
}

Judith Corte-Olivares and Gregory C. Phillips'

Department of Agronomy and Horticulture, New Mexico State University, Las Cruces, NM 88003-0003

\section{S.A. Butler-Nance}

Department of Experimental Statistics, New Mexico State University, Las Cruces, NM 88003-0003

Additional index words. Carya illinoensis, in vitro culture, propagation tissue culture

There is much interest in developing clonal propagation for pecan [Carya illinoensis (Wangenh.) K. Koch] to overcome seedling variability (Hansen and Lazarte, 1984; Knox and Smith, 1981; Merkle et al., 1987). Axillary shoots of pecan have been proliferated successfully in vitro using seedling explants (Hansen and Lazarte, 1984; Knox and Smith, 1981; Wood, 1982). The most effective of these systems resulted in a mean of one and one-half shoots per cultured node during a single culture passage, with $63 \%$ plant reestablishment success (Hansen and Lazarte, 1984). We report a procedure for propagating pecan using explants from adult trees.

Grafted 'Western Schley' trees located at two orchards southwest of Las Cruces, N. M., were used for collection of nodal explant material during two consecutive growing seasons. Specific trees were identified to represent [following the terminology of Kester (1976)] the vegetative phase (3 or 4 years old from the time of grafting), partially bearing ( 9 or 10 years old, $\approx 25 \%$ to $30 \%$ of the branches were bearing), and fully bearing

Received for publication 8 May 1989. Journal article no. 1465 of the New Mexico State Agricultural Experiment Station. We acknowledge with appreciation the technical assistance of John F. Hubstenberger. The cost of publishing this paper was defrayed in part by the payment of page charges. Under postal regulations, this paper therefore must be hereby marked advertisement solely to indicate this fact.

'To whom reprint requests should be addressed. phase (16 or 17 years old). Three collections of axillary buds were made each year during spring flush in late May, early to mid-June, and late June to early July.

Node segments 10 to $15 \mathrm{~mm}$ long with the attached axillary bud were prepared for explanting. Excised buds were kept in $1 \%$ citric acid solution until surface-sterilized by submersing briefly in $95 \%$ ethanol and for 7 min in $2 \%$ sodium hypochlorite $(50 \%$ commercial bleach) containing two drops of household detergent as a surfactant. Buds were then rinsed twice in sterile, deionized water. For surface sterilization of collections made later during the growing season, a passage through $1 \%$ benomyl solution under vacuum for $7 \mathrm{~min}$ was added after the bleach treatment, and the last rinse was in $1 \%$ citric acid.

Buds were cultured individually in $60 \times$ $15-\mathrm{mm}$ petri dishes with 100 replications (dishes) per culture treatment. The basal nutrient medium of Dunstan and Short (1977) (BDS) was used, supplemented with 0.51 $\mathrm{mM}$ ascorbic acid, $0.04 \mu \mathrm{M}$ 4-amino-3,5,6trichloro-2-pyridinecarboxylic acid (picloram), $4.4 \mu \mathrm{MN}$-(phenylmethyl) - $1 \mathrm{H}$-purin6-amine (benzyladenine, BA), and solidified with $0.8 \%$ Plant Tissue Culture agar (Carolina Biological, Burlington, N.C.). Petri dishes were randomized by assortment during incubation at $25 \pm 0.5 \mathrm{C}$ with a 16-hr photoperiod $\left(15 \mu \mathrm{mol} \cdot \mathrm{s}^{-1} \cdot \mathrm{m}^{-2}\right)$. Subcultures to fresh media were made at 30-day intervals.

Loss of replications to contamination was a severe problem, resulting in data that were not amenable to statistical analysis in five of the six collections of explants $(<20$ replications). However, in these five collections, some shoot development and multiplication was observed during the second and third culture passages from juvenile tree explants in four collections and from transitional tree explants in one collection.

Data were amenable to unbalanced replication analysis in one of the six collections made during this study, the early June collection of the second growing season $(>60$ replications), where explants of all three donor tree phases responded with shoot multiplication. A mean of one shoot per cultured bud explant was obtained from both the fully bearing and the vegetative tree materials ( $\mathrm{SE}$ \pm 0.19 and 0.17 , respectively). A maximum of six shoots per explant was observed. Explants from partially bearing trees showed significantly lower response rates $(0.3 \pm 0.12$ shoots/explant) in culture than the other donor trees. The shoots proliferated from these cultures were rooted by culture for 4 weeks on BDS medium containing $14.8 \mu \mathrm{M} \mathrm{lH}-$ indole-3-butyric acid (IBA). Normal-appearing roots formed on $40 \%$ of these shoots, resulting in complete plantlets.

The results of this preliminary study indicate potential for clonally micropropagating pecan from selected adult phenotypes.

\section{Literature Cited}

Dunstan, D.I. and K.C. Short. 1977. Improved growth of tissue cultures of the onion, Allium cepa. Physiol Plant. 41:70-72.

Hansen, K.C. and J.E. Lazarte. 1984. In vitro propagation of pecan seedlings. HortScience 19:237-239.

Kester, D.E. 1976. The relationship of juvenility to plant propagation. Proc. Intl. Plant Prop. Soc. 26:71-84.

Knox, C.A. and R.H. Smith. 1981. Progress in tissue culture methods for production of 'Riverside' stocks. Pecan Quarterly 15 :27-34.

Merkle, S. A., H.Y. Wetzstein, and H.E. Sommer. 1987. Somatic embryogenesis in tissue cultures of pecan. HortScience 22:128-130.

Wood, B.W. 1982. In vitro proliferation of pecan shoots. HortScience 17:890-891. 